\title{
Vacuum Structure of the Ichimatsu-Decomposed Lattice Models
}

\author{
K. Itoh ${ }^{\mathrm{a}}$, M. Kato ${ }^{\mathrm{b}}, \mathrm{M} . \mathrm{Murata}^{\mathrm{c}}, \mathrm{H}$. Sawanaka ${ }^{\mathrm{c}}$ and H. So ${ }^{\mathrm{c} *}$

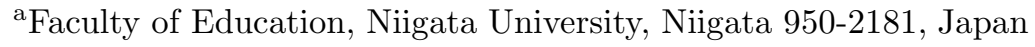 \\ bInstitute of Physics, University of Tokyo, Komaba, Meguroku, Tokyo 153, Japan \\ ${ }^{\mathrm{c}}$ Department of Physics, Niigata University, Niigata 950-2181, Japan
}

We proposed an 'Ichimatsu'-decomposed lattice gauge theory with fermionic symmetries. The vacuum structures of the gauge sector with two coupling constants $\left(\beta_{p}, \beta_{c}\right)$ are investigated for 3-dimensional $\mathrm{Z}_{2}$ and 4 dimensional $\mathrm{SU}(2)$ cases using mean-field approximation and numerical simulation. We found two phases on the $\left(\beta_{p}, \beta_{c}\right)$ phase diagram for 3 -dim. $\mathrm{Z}_{2}$ case, while the diagram is a single phase for the latter.

\section{MOTIVATION}

The lattice realization of super Yang-Mills theory has been considered very difficult though highly desirable as a non-perturbative formulation [1,2]. In our previous work and talk [3. 4], we reported the presence of exact fermionic symmetries on the 'Ichimatsu' lattice, a novel lattice. If the symmetries survive in the continuum limit, they would be related to a supersymmetry if not to the BRS symmetry.

From the equation presented shortly, we know that the fermionic symmetries cannot survive on the ordinary lattice. Therefore it is of crucial importance to study the continuum limit on the 'Ichimatsu' lattice.

\section{CELL+PIPE MODEL}

Plaquettes on the regular lattice gauge theory are decomposed into two sets:

(i) plaquettes on cell

$U_{n, \mu \nu}$ on $\left\{n_{\mu}+n_{\nu} \equiv 0(\bmod 2)\right\}$;

(ii) plaquettes on pipe

$U_{n, \mu \nu}$ on $\left\{n_{\mu}+n_{\nu} \equiv 1(\bmod 2)\right\}$.

One a $(\mu, \nu)$ plane, one finds an alternating or chequered pattern and it is called as an 'Ichi-

\footnotetext{
*Talk presented by H. So. This work was supported in part by Grants-in-Aid for Scientific Research No. 12640259 and 13135209 from the Japan Society for the Promotion of Science.
}

matsu' pattern [3,4]. The fields of the Ichimatsu lattice theory are link variables, $U_{n, \mu}$, as the gauge field, and a real staggered fermion, $\xi_{n}$, as the fermion field.

The gauge action of our model (cell+pipe) is

$S_{\text {gauge }}=-\frac{\beta_{c}}{2} \sum_{\text {on cell }} \operatorname{Tr} U_{n, \mu \nu}-\frac{\beta_{p}}{2} \sum_{\text {on pipe }} \operatorname{Tr} U_{n, \mu \nu}$.

The fermionic symmetry transformation of the fermion field is written as

$\delta \xi_{n} \sim\left(1-\frac{\beta_{p}}{\beta_{c}}\right) \sum_{0<\mu<\nu}(-)^{n_{\mu}+n_{\nu}}\left(C_{n, \mu \nu}^{(+)}+C_{n, \mu \nu}^{(-)}\right) F_{n, \mu \nu}$

where $C_{n, \mu \nu}^{ \pm}$are Grassman-odd parameters.

It should be emphasized that on the usual regular lattice $\left(\beta_{c}=\beta_{p}\right)$, the transformation goes down to $O(a)$. Therefore it is important to study the dynamical properties on the twocouplings space $\left(\beta_{p}, \beta_{c}\right)$. From now on, we switch off fermion effects in our model and concentrate on the vacuum structure of the gauge sector.

\section{EXPECTED PROPERTIES OF OUR MODEL}

\subsection{Mean Field Approximation}

We take a link variable $\left\langle U_{n, \mu}\right\rangle=v_{\mu}$ as a mean field. This approximation is not gaugeinvariant and it is useful only in the first view of the phase diagram. 
The results are

(1) There exists an exact duality under $\left(\beta_{c}, \beta_{p}\right) \leftrightarrow\left(\beta_{p}, \beta_{c}\right)$, that is, the partition functions are invariant under the interchange of couplings;

$$
Z_{\mathrm{MF}}\left(\beta_{c}, \beta_{p}\right)=Z_{\mathrm{MF}}\left(\beta_{p}, \beta_{c}\right) .
$$

(2) In the cell model (defined by putting $\beta_{p}=$ $0)$ and the pipe model $\left(\beta_{c}=0\right)$, phase transitions occur.

These results are independent of the spatial dimension and the gauge group. A rough sketch of the diagram is shown in Fig. 1.

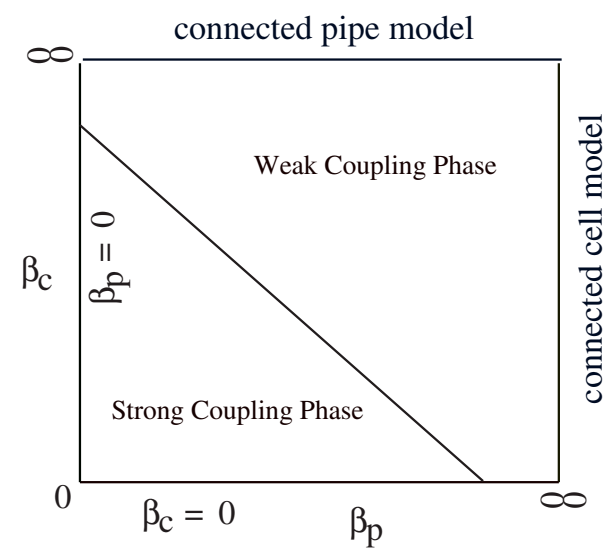

Fig. 1. Phase diagram of cell+pipe model in mean field approximation.

\subsection{Analytical Results}

1. Regular lattice gauge theory $\left(\beta_{c}=\beta_{p}=\beta\right)$

When $\beta_{p}=\beta_{c}=\beta$, our model becomes a usual regular lattice gauge theory. On the regular lattice, the 3D $Z_{2}$ gauge theory has a phase transition point while the $4 \mathrm{D} \mathrm{SU}(2)$ gauge theory has a crossover point.

2. Four phase boundaries

In order to understand the phase diagram, it is necessary to analyse the limiting cases, $\beta_{c}$ or $\beta_{p} \rightarrow 0$ or $\infty$. We can obtain analytical results for these limiting cases. In the cell model, $\beta_{p}=0$, the system is decomposed to a finite degrees of freedom and it is exactly solvable. The pipe model, $\beta_{c}=0$, is equivalent to one plaquette model and is also exactly solvable. Both cases have no phase transition contrary to the result of the mean field approximation.
In $\beta_{p}$ or $\beta_{c} \rightarrow \infty$, plaquettes on cell or pipe are strongly connected. The dynamics for these situations is not known and the numerical analysis is necessary.

3. Possibility of the continuum limit and its phase transition

To take the continuum limit of our model, we must avoid the phase transition point or line. If we go across the singularity, then we miss the information on the vacuum, such as confinement. If one want to be consistent with confinement and asymptotic freedom, the phase diagram must be smoothly connected between the weak and strong coulping regions.

\section{PHASE DIAGRAM FOR ICHIMATSU PATTERN LATTICE}

\subsection{D $\mathrm{Z}_{2}$ Case}

The action is written as

$$
S=-\beta_{c} \sum_{\text {plaq.on cell }} U U U U-\beta_{p} \sum_{\text {plaq.on pipe }} U U U U
$$

where $U$ is a link variable taking the values, \pm 1 . Note the normalisation of the coupling constants differs from the $\mathrm{SU}(2)$ case. For the regular lattice $\left(\beta_{c}=\beta_{p}=\beta\right)$, it is found that there occurs the 2 nd order phase transition at $\beta=0.76$ obtained by the analysis of the equivalent Ising spin. We have simulated $3 \mathrm{D} \mathrm{Z}_{2}$ cell+pipe model for the size, $4^{3}, 8^{3}$ and $16^{3}$.

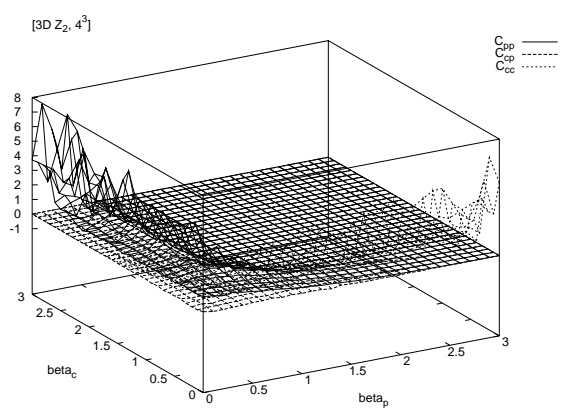

Fig. 2. $3 \mathrm{D}$ plot for $\left(\beta_{p}, \beta_{c}\right.$, specific heat) in $3 \mathrm{D}$ $\mathrm{Z}_{2}$ model.

From Fig. 2, we can see that the phase transition point is extended to the connected pipe $\left(\beta_{c} \rightarrow \infty, \beta_{p}=0.55\right)$ model and the connected cell $\left(\beta_{p} \rightarrow \infty, \beta_{c}=0.38\right)$ model. 
The phase diagram for $3 \mathrm{D} \mathrm{Z}_{2}$ model is obtained in Fig. 3.

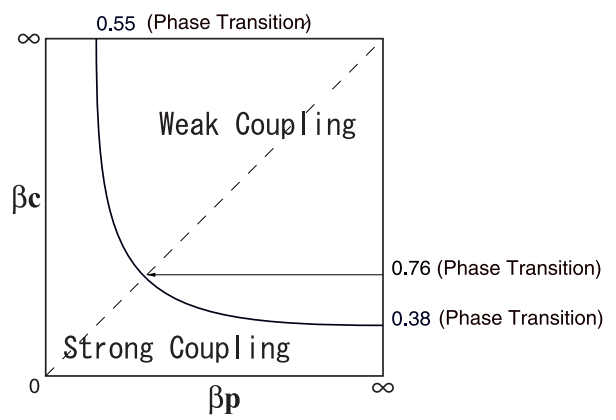

Fig. 3. Phase diagram for $3 \mathrm{D} \mathrm{Z}_{2}$ model.

\section{2. $4 \mathrm{D} \mathrm{SU}(2)$ case}

For lattice size, $8^{4}, 12^{4}, 16^{4}$, we calculated the internal energy and the specific heat. In Fig. 4a, we show a crossover behaviour $\left(\beta_{p}=\beta_{c} \sim 2.0\right)$ on a regular lattice and a sharp peak of the specific heat for the connected cell model in Fig. 4b.

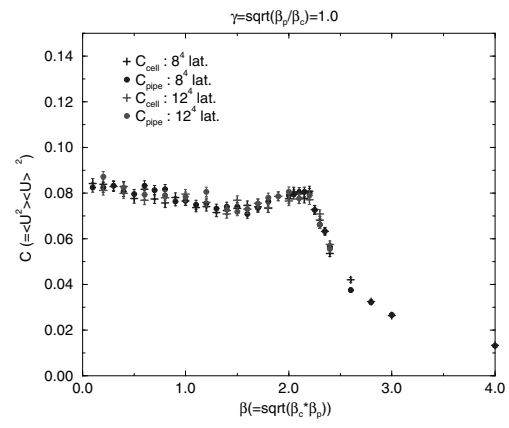

Fig. 4a. A crossover in the regular lattice model.

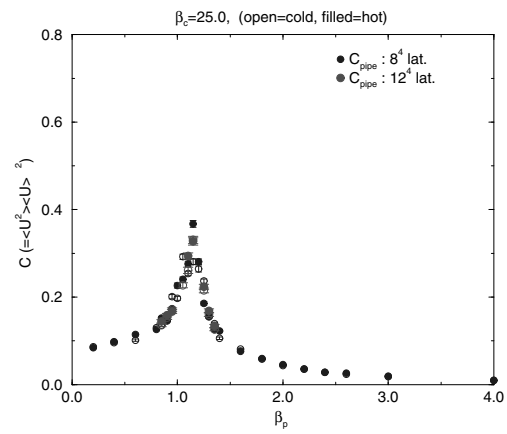

Fig. 4b. A peak of specific heat at $\beta_{c}=25$.

Our simulations are consistent with the following statements: 1) the peak in Fig. 4b is that of the 2nd order phase transition; 2) no peak is present in the connected cell model; 3) a crossover line extends to $\left(\beta_{p} \rightarrow \infty, \beta_{c} \sim 0\right)$.

\section{SUMMARY AND DISCUSSIONS}

To consider exact fermionic symmetries on lattice, we decomposed all plaquettes into two sets, the cell and pipe. The vacuum structure of the gauge sector was investigated to study its continuum limit.

Phase diagrams of $3 \mathrm{D} \mathrm{Z}_{2}$ and $4 \mathrm{D} \mathrm{SU}(2)$ models were studied. We found a 'corridor' (singularity free region) between strong coupling and weak coupling regions for $4 \mathrm{D} \mathrm{SU}(2)$ case (Fig. 5); the diagram is a single phase. The $3 \mathrm{D} \mathrm{Z}_{2}$ model has two phases.

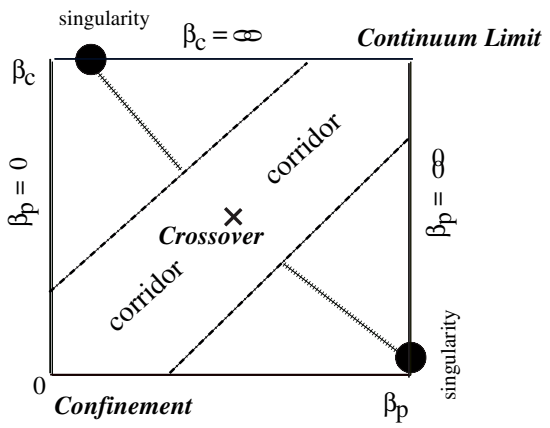

Fig. 5. Phase diagram for 4D SU(2) model.

This is our first non-perturbative result on an 'Ichimatsu' lattice gauge theory. Further results along this direction will be reported in the forthcoming paper 5 .

\section{REFERENCES}

1. G. Curci and G. Veneziano, Nucl. Phys. B292 (1987) 555.

2. D. B. Kaplan and M. Schmaltz, Chin. J. Phys. 38 (2000) 543.

3. K. Itoh, M. Kato, H. Sawanaka, H. So and N. Ukita, Prog. Theor. Phys. 108 (2002) 363.

4. K. Itoh, M. Kato, H. Sawanaka, H. So and N. Ukita, Fermionic Symmetry in IchimatsuDecomposed Lattice Models, in this volume.

5. K. Itoh, M. Kato, M. Murata, H. Sawanaka and $\mathrm{H}$. So, in preparation. 